\title{
1.8-mm coaxial microincision cataract surgery with the Stellaris Vision Enhancement System
}

\author{
Ioannis Mallias, Panagiota Mylova, Anastasia Tassiopoulou \\ Laser Plus Eye, Nea Smyrni, Athens, Greece
}

\begin{abstract}
The purpose of this review is to analyse the benefits of phacoemulsification with $1.8-\mathrm{mm}$ coaxial microincision cataract surgery using the Stellaris Vision Enhancement System. The Stellaris operating system and its advantages are explained and analysed. Other techniques are also referred to. The surgical technique of 1.8-mm microincision cataract surgery using Stellaris is described and some essential and helpful tips are explained. The Stellaris Enhancement System makes 1.8-mm microincision cataract surgery fast and easy for the surgeon, leading to high rates of patient satisfaction from the first postoperative days.
\end{abstract}

KEY WORDS: cataract surgery, 1.8-mm cataract surgery, 1.8-mm C-MICS, Stellaris Vision Enhancement System

Ophthalmol J 2017; Vol. 2, No. 2, 54-57

\section{ADVANTAGES OF COAXIAL MICROINCISION CATARACT SURGERY (C-MICS)}

Phacoemulsification techniques have evolved over the years. Evolution in technology has allowed surgeons to create progressively smaller incisions. Going from the original $3.5-\mathrm{mm}$ procedure to the $2.8-\mathrm{mm}$ procedure was a major step forward, and many surgeons questioned the need to go even smaller. However, there is evidence showing significant advantages of the sub-2-mm procedure. Some of them are reduction of the following: inflammation $[1,2]$, corneal oedema $[2,3]$, endothelial cell loss [4], and surgically induced astigmatism $[3,5]$. Also, there is an improvement in corneal wound strength [6] and fluidics efficiency [7].

There are many surgeons who have adopted other techniques such as biaxial/bimanual microincision cataract surgery (B-MICS) popularised by Jorge Alio and others. However, some surgeons find B-MICS more difficult or less efficient that their customary $2.8-\mathrm{mm}$ cataract surgery and return to their previous technique. Others have embraced the $1.8-\mathrm{mm}$ coaxial microincision cataract surgery (C-MICS) procedure, introduced with the Stellaris
Vision Enhancement System for phacoemulsification (Fig. 1).

The 1.8-mm C-MICS offers the benefits of a sub-2-mm procedure with little or no learning curve. It is compatible with any technique, and surgeons' familiar fluidics settings can be utilised. In contrast with B-MICS, $1.8-\mathrm{mm}$ C-MICS maintains the infusion sleeve to improve chamber stability, reduce leakage, protect the cornea from friction and stress, and maintain a water-tight seal. The non-dominant hand is not "stuck" inside the eye to maintain infusion, and smaller, more ergonomic side port instruments can be used.

$1.8-\mathrm{mm}$ C-MICS is especially helpful in cases with small pupils, IFIS cases, situations with disrupted zonules or capsule, pseudoexfoliation. The smaller incision is also a benefit when operating on patients that have previously undergone radial keratotomy (RK) or penetrating keratoplasty (PK) because it allows the surgeon to work between the $\mathrm{RK}$ incisions or the PK sutures. There is also early evidence [8] that C-MICS may provide improved safety as far as capsule tears are concerned. 


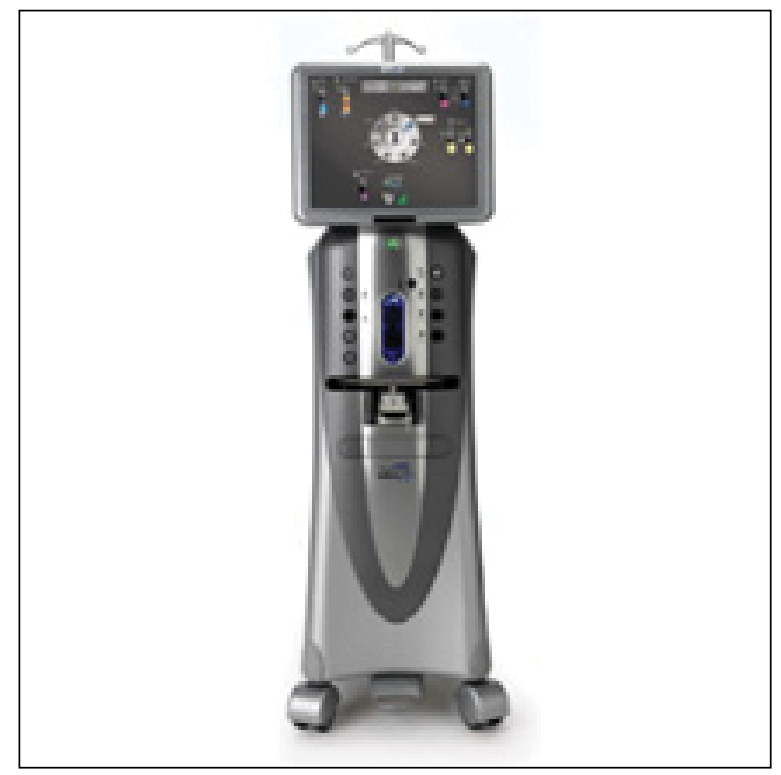

FIGURE 1. Stellaris Vision Enhancement System for phacoemulsification

\section{FLUIDICS}

When it comes to fluidics, the safety of Stellaris is quite simple. Inflow must always replace any outflow through the needle and any leakage through the incisions. The anterior chamber only contains approximately six drops of fluid, which means that if outflow exceeds inflow by six drops the anterior chamber will collapse, causing damage in many intraocular tissues. With sub-2-mm procedures there is advanced difficulty when it comes to fluidics control because of the smaller infusion instruments for B-MICS or the smaller infusion sleeves for C-MICS, which deliver less fluid per second for any given bottle height. To surpass this limitation, one alternative is to increase the bottle height in order to increase the potential infusion volume per second. The problem with this approach is that IOP is directly proportional to the bottle height and inversely proportional to the outflow. To overcome these problems, Stellaris has been introduced to an automated infusion option called DigiFlow.

DigiFlow technology pressurises the infusion bottle with a digitally controlled air pump. The surgeon sets the actual bottle height and then a precise amount of additional air pressure can be selected to achieve the desired effective bottle height. This has practical value for surgeons who want bottle heights greater that their operating room ceiling. A field observation evaluation (FOE) has been conducted by the manufacturer [9], which included 55 surgeons from the USA, Spain, and India. $76 \%$ of the partic- ipants reported improved chamber stability with the DigiFlow compared to the gravity feed for the same equivalent bottle heights.

When designing the Stellaris for fluidics safety with $1.8 \mathrm{~mm}$ C-MICS, a decision was made to engineer each component of the system to work synergistically to optimise chamber stability. The needle and sleeve were designed as a balanced pair using computational fluid dynamics, computer-aided design, and finite element analysis computer modelling.

The Stellaris Attune handpiece was designed with a 50\% larger infusion channel to deliver more BSS per second at any given bottle height. Stellaris tubing was created with a large diameter and high compliance infusion line, and a smaller diameter and low compliance aspiration line. This combination optimises the ability to deliver BSS, stabilises the anterior chamber, and minimises post-occlusion surge for vacuum settings up to $300 \mathrm{~mm} \mathrm{Hg}$.

\section{CHAMBER STABILITY-STABLE CHAMBER TUBING}

When it comes to higher vacuum levels up to $600 \mathrm{~mm} \mathrm{Hg}$, Stable Chamber tubing was developed (Fig. 2). Stable Chamber tubing incorporates a section of flexible tubing, connecting the handpiece to a filter, maintaining in this way the natural flexibility and feel of standard tubing. The tubing that connects the filter to Stellaris has low compliance and a smaller internal diameter, which increases resistance to outflow and further reduces the potential chamber instability and post-occlusion surge. The filter captures nuclear fragments and prevents the smaller tubing from clogging. Stellaris pump technology now incorporates a Stable Chamber Fluidics Module. For surgeons who prefer high vacuum techniques, $1.8-\mathrm{mm}$ C-MICS can safely be performed with the Stable Chamber tubing (as described above). The advantage of using $600 \mathrm{~mm} \mathrm{Hg}$

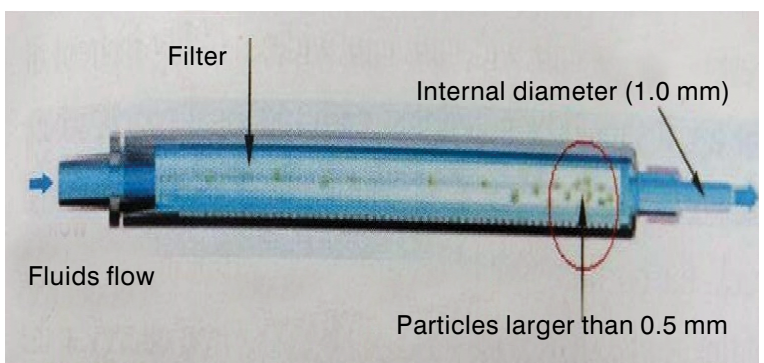

FIGURE 2. Stable Chamber tubing 


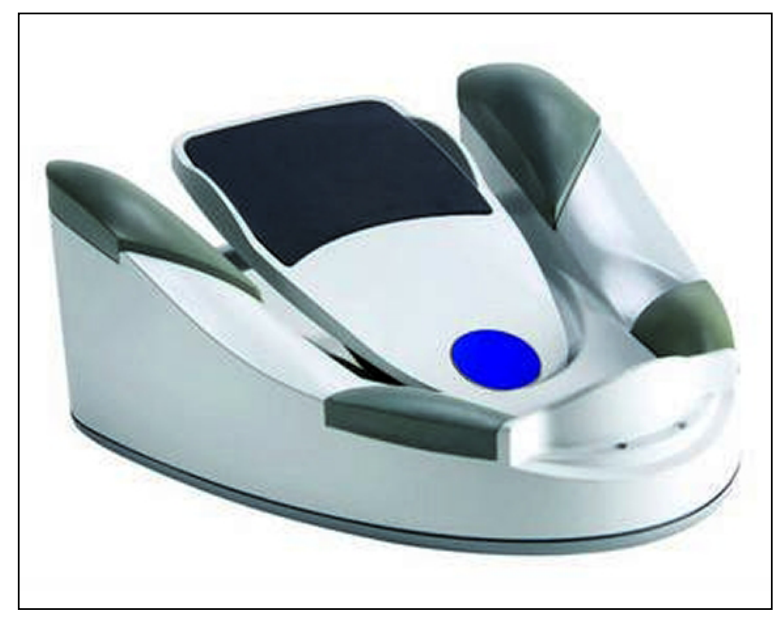

FIGURE 3. Dual linear foot pedal

with Stable Chamber is only obvious when the tip is occluded. At that time, flow stops and vacuum migrates to the tip, with $600 \mathrm{~mm} \mathrm{Hg}$ vacuum holding force to facilitate chopping or quadrant removal. Then, as the nucleus is aspirated, the eye is protected from high vacuum surge by the flow restriction of Stable Chamber tubing.

\section{FLUIDICS AND MACHINE SETTINGS}

Another fluidics advance for Stellaris is the wireless, Bluetooth, dual linear foot pedal (Fig. 3). It has a variety of programmable options and can be used like a traditional phaco foot pedal only for the updown or pitch direction. The surgeon can program two or more low and high phaco settings and manually switch between them on a panel. Most users find a significant advantage in utilising the dual linear function where power can be controlled in either up and down "pitch" direction or horizontal "yaw" direction. The vacuum can be programmed to be controlled in the other direction.

The difference between a standard foot pedal and a dual linear foot pedal is like the difference between driving a car with cruise control and using an accelerator pedal. On a straight highway with stable conditions cruise control is convenient, but on a winding mountain road the driver will need to speed up or slow down to match the changing conditions. When using a dual linear foot pedal, one can use very low flow and followability when working close to the iris or capsule and increase the followability when starting to work more centrally in a safe position. In this way, a higher level of safety and control is offered to the user compared to a machine with a single function foot pedal. This trilinear function facilitates phacoemulsification with any case using any technique, but is particularly valuable when dealing with small pupils, IFIS cases with floppy iris, and cases with capsule tears or broken zonules.

\section{POWER MODULATION OPTIONS-THERMAL EFFECTS}

All major manufacturers have incorporated various power modulation options with different trade names into their equipment. Stellaris was designed with the "Attune Energy Management System" that allows "on" and "off" times with pulse rates as low as 2 milliseconds and up to 250 pulses per second, as well as surgeon-programmable duty cycles. Stellaris also offers a variety of power modes such as pulse, burst, multi-burst, fixed burst, and continuous power. Additionally, it offers a choice of traditional "square wave" pulses or "waveform" pulses.

Waveform pulses have the power ramp up gradually over the course of the selected "on" time, then they reach the selected power limit and drop abruptly back down towards baseline, but not to zero power. Square waves have the power spike abruptly up to the selected power limit, plateau for the selected "on" time, and then drop steeply back down to base line. Waveform pulses include less initial repulsive force because the power ramps up more slowly to improve followability, and less total energy per pulse for identical power limits and duty cycles, compared to the square wave pulses.

The clinical significance of properly designed power modulation has become more evident with the increase of reports [10-12] of corneal wound burn. Minimising thermal effects during phaco procedure should become more important as the incision size decreases and incision leakage is minimised, to improve chamber stability. In a paper presented by M.E. Schafer, PhD it was found that Stellaris had the lowest absolute temperature rise and was the most consistent in terms of cooling of the tip region [13]. These results are consistent with clinical outcomes [14]. Stellaris was found to have the lowest incidence of wound burns.

\section{C-IMICS TECHNIQUE}

When it comes to surgical technique, C-MICS is compatible with any chopping, divide and conquer, phaco flip, or other variation a surgeon chooses. The 
only learning curve is performing the continuous curvilinear capsulorhexis through the smaller incision. The standard Utrata forceps did not open widely due to the size of the incision, so now there are several designs available (disposable as well) through a variety of companies including Stellaris' manufacturer.

What should be noted is that the proper incision size is of high importance. The smaller infusion sleeves may be thinner and more easily crimped if the incision is too tight. There are two points regarding the sleeves. The sleeve is designed for the infusion to be directed laterally at approximately $30-40^{\circ}$ through each infusion side ports. If the sleeve is positioned too far, back from the front flair of the needle, excess infusion will be directed forward, reducing followability and pushing lens material away from the tip. The correct position is for the front edge of the sleeve to slightly overlap the wider front portion of the needle. The surgeon can verify the correct sleeve placement before entering the eye by engaging foot pedal position one (infusion) and making sure that the majority of fluid is directed laterally.

With the smaller incision and somewhat thinner sleeve, it is easier to insert using reverse flow through the needle. The reverse flow immediately inflates and lubricates the corneal tunnel and pressurises the anterior chamber before the sleeve is introduced.

In conclusion, Stellaris was engineered to balance multiple technologies in order to ensure safety and efficiency for sub-2-mm C-MICS and B-MICS. The design offers these same advantages for traditional $2.8-\mathrm{mm}$ techniques and can easily be adapted to go below $1.8 \mathrm{~mm}$ for C-MICS as lens technology and surgeon preferences evolve. At this time, a combined phaco and posterior segment Stellaris is available.

\section{REFERENCES}

1. McDonald JE. Comparison of postoperative flare/cells after microincision cataract surgery compared to small incision cataract surgery. Presented at ASCRS Symposium on Cataract, IOL and Refractive Surgery.

2. Han $Y$, Wang J. [New progresses in micro-incision cataract surgery]. Zhonghua Yan Ke Za Zhi. 2012; 48(4): 369-373, indexed in Pubmed: 22800458.

3. Braga-Mele R. Is smaller truly better? An evaluation of phaco incision size and astigmatism. Presented at AAO Annual meeting. San Francisco, 2009.

4. Zafirakis P. Stellaris phaco platform versus Infinity torsional phaco mode. Presented at ASCRS Annual meeting. San Francisco, 2009.

5. Heng WJ. Surgically induced astigmatism in standard vs microincision coaxial phacoemulsification. Presented at the 11 th Conference of the China Cataract Society in Xi'an China, 2008.

6. Barrett GD. Minimizing astigmatism and improving wound security. Presented at Asia-Pacific Academy of Ophthalmology, 2009.

7. Hunkeler JD. Comparison of BSS usage between Stellaris MICS and Infinity intrepid. Presented at ASCRS Annual Meeting. Boston, 2010.

8. Agarwal A, Agarwal A, Jacob S. Phacoemulsification. Jaypee Brothers Medical Publishers (P) Ltd., Panama City 2011: 227-235.

9. Bausch\&Lomb. Pressurized infusion field observation study \#606;2009.

10. Belkin A, Abulafia A, Michaeli A, et al. Wound temperature profiles of coaxial mini-incision versus sleeveless microincision phacoemulsification. Clin Exp Ophthalmol. 2017; 45(3): 247-253, doi: 10.1111/ ceo.12851, indexed in Pubmed: 27731924.

11. Hamza I. Wound burn in MICS with OZil Custom Pulse Mode. Presented at 2008 ASCRS film festival, Chicago, IL, 2008, April 4-9.

12. The FDA "Manufacturer and User Facility Device Experience" (MAUDE) database. http://www.accessdata.fda.gov/SCRIPTS/cdrh/cfdocs/ cfmaude/Search.cfm.

13. Schafer ME. Thermal response of phacoemulsification tips in normal and occluded conditions. Presented at the ESCRS Congress. Barcelona, 2009, Sept. 15.

14. Olson RJ, Chan C, Bradley M, et al. An Analysis of Wound Burns in the US and Canada. Poster presented at ASCRS Symposium on Cataract, IOL and Refractive Surgery. Boston, MA, 2010. 